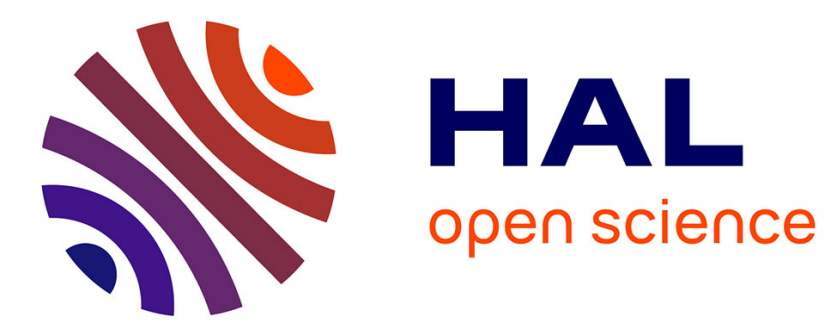

\title{
Milieux sociaux et genèse de la personne selon René Zazzo
}

Philippe Malrieu

\section{To cite this version:}

Philippe Malrieu. Milieux sociaux et genèse de la personne selon René Zazzo. Enfance, 1996, 2, pp. 134-142. halshs-01076977

\section{HAL Id: halshs-01076977 https://shs.hal.science/halshs-01076977}

Submitted on 23 Oct 2014

HAL is a multi-disciplinary open access archive for the deposit and dissemination of scientific research documents, whether they are published or not. The documents may come from teaching and research institutions in France or abroad, or from public or private research centers.
L'archive ouverte pluridisciplinaire HAL, est destinée au dépôt et à la diffusion de documents scientifiques de niveau recherche, publiés ou non, émanant des établissements d'enseignement et de recherche français ou étrangers, des laboratoires publics ou privés. 


\section{Milieux sociaux et genèse de la personne selon René Zazzo}

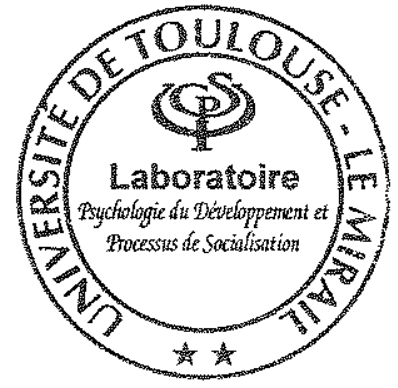

"L'intelligence n'est rien d'autre peut-être que la structure du monde qui prend conscience d'elle-même. " $\mathrm{R}$. Zazzo ecrit ces mots dans la préface à son deuxième ouvrage, composé dans les heures où il partageait son temps entre la recherche scientifique et la lutte contre le nazisme. Marquant ainsi sa conviction rationaliste: il n'y a dans le monde aucune réalité qui reste étrangère à une approche par l'intelligence. Cette conviction s'appuyait sur une réflexion épistémologique qui prolongeait les débats du début du siècle sur la possibilité d'instaurer une psychologie scientifique. Et Zazzo assurément prend position contre les philosophies qui déniaient aux méthodes scientifiques le droit d'accéder a la réalité psychique, contre la conception bergsonienne de données immédiates de la conscience, contre Husserl qui fait de lintention le processus fondateut de la structuration du monde connaissable, et par là mis à l'abri de toute explication à partir de conditions extérieures. Science, la psychologie aux yeux de Zazzo récuse l'introspection pour son subjectivisme, elle doit assumer la fonction de «tendre compte du fait en le ramenant à ses conditions d'existence : les facteurs biologiques et sociaux $»^{1}$. Mais "rendre compte» pour lui passe par deux exigences, dont la deuxième a été méconnue par certains promoteurs de la psychologie scientifique.

La première concerne la recherche des conditions du fait dans une situation donnée, d'un point de vue «statique» si l'on peut dire (terme impropre puisque la réaction psycbique ne va pas sans une transformation du milieu et de l'organisme). Et elle concerne aussi l'etude de la construction de cette réaction au cours de l'enfance, ou dans les temps longs d'une vie, de plusieurs générations, ou encore de l'évolution de l'espèce. A ce niveau, une préoccu-

* Département de psychologie, Université de Toulouse - Le Mirail, 31400 Toulouse.

1. La rationalité en psychologie, in Conduites et conscience, t. II, 1968, p. 38-39. 
pation essentielle selon Zazzo sera d'explorer les relations entre facteurs biologiques et sociaux. Ainsi voit-on que les facteurs culturels ont une prise variable sur l'individu en fonction du développement de ses structures biologiques - comme le montrent les études sur la débilité ${ }^{1}$.

Mais il y a une deuxième exigence pour la psychologie scientifique. Elle ne peut se limiter à expliquer l'homme dans ses conditions d'existence, «qui peuvent l'aliéner ou le révéler a lui-même, c'est-à-dire en faire un objet ou un véritable sujet». Elle doit l'expliquer aussi «dans ses raisons d'être, c'estù̀" dire dans un systeme de fins et de valeurs... comprendre l'homme par ses ori gines et par ses buts, depuis ses hérédités les plus strictes... jusqu'à cette exigence d'autonomie, de liberté, qui fait de lui une personne $\aleph^{2}$.

Conditions-raisons d'être: il ne s'agit pas d'une part de causes efficientes, de l'autre de causes finales. La recherche des raisons d'être vise à définir des «significations, par exemple l'adaptation de l'être humain dans un univers de valeurs». Il ne s'agit plus de «ramener» le fait aux passés qui le conditionnent, mais de s'interroger sur sa fonction dans les totalités où il se produit, et dans l'avenir sur lequel il ouvre. Ces raisons sont chez l'homme découvertes par des activités conscientes qui délibèrent sur des valeurs: activités libres. «Sans conscience et sans liberté le sujet n'est rien... Nier ma liberté, nier mon évidence, j'en suis libre, mais c'est manque de sincérité par excès de rigueur intellectuelle. » Zazzo refuse d'admettre en lui deux règnes distincts, « celui de la nécessité, celui de la liberté, celui-ci échappant à ma raison», il propose aux psychologues «d'entreprendre scientifiquement une recherche de la liberté», sans se dissimuler la difficulté de l'entreprise'.

C'est de la méthode qui lui permet de la réaliser que nous voudrions évoquer quelques aspects. Non sans avoir rappelé que ce fut chez lui une préoccupation constante. On la retrouve en 1982 dans sa contribution à La psychologie de demain, où il prévoit que la psychologie de l'enfant, après les analyses fonctionnelles qui ont fondé la psychologie génétique, voudra saisir l'enfant « dans son unité, son indivisibilité »: l'individu sera « objet de science $»^{4}$. S'il met en évidence l'importance des découvertes sur les compétences innées du nourrisson, il précise aussi que c'est en s'inspirant de la méthode dialectique pratiquée par Wallon qu'il sera possible d'atteindre la spécificité des conduites humaines. Elle est attentive aux contradictions auxquelles est exposé l'enfant (entre la tram dition et son expérience, entre le formalisme du langage et la luidité des données sensibles, entre le réel et ses représentations). C'est en abordant ces contra-

1. Les déterminants de la débilité sont biologiques, mais l'insuffisance n'est pas telle qu'elle interdise l'adaptation à certaines exigences du milieu social (Les débilités mentales, Paris, 1969 , p. 27-33).

2. Pour une psychologie intégrale, Conduites et conscience, II, p. 11 (1968, le texte est de 1963).

3. La rationalité en psychologie, loc. cit., p. 39-41.

4. L'enfance de la psychologie de l'enfant et son devenir, in La psychologie de demain, sous la direction de P. Fraisse, 1982, p. 167-189. 
dictions que le sujet construit ses capacités, en « participant à des ensembles où il devrait trouver des raisons d'exister » (souligné $\mathrm{P}$. M.) : des capacités qui le rendent autonome, non en le soustrayant à ses conditions biologiques et sociales d'existence, mais en les inscrivant dans de nouveaux milieux qui en resignifient les régulations premières.

Sa recherche sur tes jumeaux identiques a permis à Zazzo de s'inscrire dans cette perspective : les déterminants de l'hérédité se trouvent contenus et déportés par la participation des jumeaux à des activités où ils trouvent les motivations d'une recherche de singularisation. Mais nous retiendrons d'abord les apports de la recherche collective sur le développement des garçons de 6 à 12 ans à la définition de la méthode dialectique utilisée par Zazzo et ses collaborateurs!

L'intention première en était d'étudier scientifiquement le cheminement vers «le système de valeurs et de liberté», vers les attitudes de la personne "centre de responsabilité, conscience d'autonomie» (p. 10) au cours de l'en. fance. Les composantes biologiques n'en étaient pas méconnues. En un premier temps, l'équipe voulut réduire autant que possible les facteurs sociaux des différences interindividuelles, et constituer des groupes socioculturels homogènes: en chacun d'eux la variance pouvait être attribuée, plus fortement que dans une population tout venant, aux facteurs biologiques (bien qu'une surdétermination par le milieu ne soit pas exclue). Pour chaque variable prise isolément (dynamisme évolutif, hiérarchie des valeurs, anxiété par exemple) on pouvait alors espérer atteindre des lois générales de développement de 6 à 12 ans.

Quelle est alors l'influence des milieux socioculturels sur ces lois? Comment le développement est-il freiné, accéléré, orienté par l'appartenance des enfants à ces milieux? R. Zazzo en marque fortement le caractère dialectique dans des Réflexions sur la notion de milieu (p. 121-132) et dans les conclusions à son étude sur l'échelle de développement psychosocial (p. 360-368).

Déjà la position de chaque enfant dans ses milieux socioculturels est définie par une combinaison de caractéristiques susceptibles de faire saisir les déséquilibres auxquels il est exposé, avec lesquels il peut jouer. Entrent en compte en effet les professions des deux parents et des grands-parents, leur niveau d'études, ce qui permet des confrontations entre les deux dimensions, l'établissement de milieux multiples. La cote sociale ainsi établie (chap. 1, par M. C. Hurtig et B. Zazzo) peut révéler des instabilités latentes. Car pour $\mathbb{R}$. Zazzo et son équipe, le milieu social ne peut être conçu comme un cadre impératif de développement. Il n'y a pas pour l'enfant un modele de perfection qui serait fourni par les adultes, par les parents, l'école, la société globale: il y a une pluralité de modeles. Et encore faut-il prendre en compte qu'ils changent d'une génération à l'autre, et que l'enfant est sensible à ces changements, aux conflits qui se manifestent entre ces milieux. 
Zazzo peut donc inscrire les rapports milieux enfants dans une vision dialectique:

$1 /$ «L milieu n'existe que dans la mesure où l'organisme l'assimile, et cette assimilation transforme du même coup l'organisme et le milieu. 》 Il dépend de la réceptivité et de la réactivité de l'individu que les milieux socioculturels l'influencent comme ils le font : et elles dépendent elles-mêmes de ses structures biologiques et de la façon dont il est atteint par les conflits qui existent entre ses milieux (famille, sa situation économique, son idéologie, école, société globale).

2/L'influence de chaque milieu ne s'exerce pas indépendamment de celle des autres: il y a des structures de milieux, qui en un sens sont imposées par "histoire de la société, elles sont « objectives», mais les individus se situent de façon originale dans les réseaux qu'elles constituent, et qu'ils contribuent à transformer.

$3 /$ L'influence des milieux varie selon les secteurs évolutifs (organisation somatique, intelligence, affectivité, sociabilité...) : entre les diverses caractéristiques d'un milieu, il faut évaluer le poids de chacune d'entre elles sur le secteur évolutif considéré, en prenant en compte qu'il varie en fonction de l'accès de l'individu à des conduites nouvelles, à des milieux nouveaux.

$4 /$ Il y a des hétérochronies dans le milieu en raison de la coexistence des générations successives. Chaque génération est, représente une époque, elle vit son milieu d'une façon différente des autres générations. Dans la famille par exemple, il y a «les strates, les paliers en profondeur de plusieurs générations - séquelles actives du passé, influences des grands-parents dans le statut socioculturel et la mentalité des parents, effets de leur promotion culturelle, apports des enfants eux-mêmes... (car) - ils sont des récepteurs plus sensibles à certaines influences de leur temps; antennes tendues vers le monde extérieur, vers les innovations techniques et culturelles, ils transforment leur entourage immédiat, du moins dans une certaine mesure » (p. 129-131).

Cette conception dialectique des rapports individu-milieu ouvre la voie à une théorie de la personnalité et de la personne: «Le milieu, c'est ce système de déterminations réciproques, où les générations accordent leurs désaccords, où les décalages composent un équilibre instable et dynamique. Les décalages, les tensions internes, définissent le milieu en tant que système d'interrelations temporelles, mais ils rendent compte aussi de son dynamisme, du ressort de son évolution propre» (p. 132).

On peut dire: le milieu ainsi défini est le champ qui appelle de la part de l'individu des «réactions de situation》 de ses diverses composantes les unes par rapport aux autres. Ces réactions sont de niveaux divers: elles vont depuis l'adaptation du corps propre à ses milieux jusqu'aux élaborations cognitives scientifiques. Ne pourrait-on pas pour les définir reprendre ce que dit Zazzo des régulations psychologiques: "L'ensemble des processus qui assurent les rapports de l'organisme avec les situations changeantes du milieu, le psychisme défini comme fonctions de maîtrise du monde extérieur et d'adaptation.» Il en résulte une forme d'autonomie, «une libération du 
milieu pour en fin de compte $s^{\prime} y$ intégrer mieux, et le mâ̂triser $\rangle^{1}$. Ne pourrait-on dire qu'à ce niveau d'autonomie nous sommes en présence de la régulation des «conditions d'existence, facteurs biologiques et sociaux», dont l'exploration est la première tâche de la psychologie? Et parler de personna. lité à son sujet?

Mais R. Zazzo distingue personnalité et personne. Il pense qu'en dépit de la proximité de sens des deux termes, le mot de personnalité désigne « une réalité d'ordre empirique, celui de personne, une valeur une notion d'ordre ontologique: c'est la singularité irréductible dans l'univers des semblables $\gg{ }^{2}$. Se plaçant au niveau des valeurs, il donne à la notion de personne un double contenu: elle est l'être à respecter dans un monde de personnes (aspect social); et elle est le lieu de la prise de conscience par le sujet de son autonomie: «Appropriation de soi qui s'accomplit dans l'appropriation d'une échelle de valeurs» (ibid.) : aspect moral, concernant la liberation par le sujet, évidemment social, des fatalités que peuvent être pour lui le legs héréditaire ou les institutions qui aliènent ses capacités d'invention. Or cette libération, acte de personne, passe par des conduites affectives (de révolte ou d'admiration par exemple) et cognitives (intelligence des situations, analyses des origines des frustrations, construction d'un projet), qui ne sont pas d'une autre nature que celles qui permettent l'adaptation au monde, et que nous plaçons dans le cadre de la personnalité. Ce recoupement des deux notions explique qu'on puisse prendre l'une pour l'autre.

Pourtant Zazzo tient à les distinguer, et ce nous paraît être un de ses apports essentiels à une compréhension exacte de la fonction de la psychologie dans l'ensemble des sciences humaines. On peut situer cette distinction par rapport à sa conception dialectique du poids des facteurs génotypiques, mésologiques et individuels dans les divers types de conduites.

1/L'influence de l'hérédité varie en fonction inverse de la complexité des conduites: "Le rapport entre facteurs génotypiques et non génotypiques reste à peu près stable au cours de la vie pour les fonctions mentales plus simples, il se modifie au bénéfice des facteurs non génotypiques pour les plus complexes $»$ (Les jumeaux, p. 540).

$2 /$ Les influences des milieux socioculturels traduisent les processus d'inscription des conduites primitives dans les cadres culturels (techniques, politiques, scientifiques, idéologiques...) qui se sont constitués au cours de l'histoire des sociétés. Et Zazzo renvoie à l'exemple de la mentalité dite primitive et de la mentalité logique de notre civilisation. Dans les relations de l'individu à son entourage, il construit ses conduites en fonction des incitations de celui-ci, et il en résulte de nouvelles inter-structurations des fonctions au cours de l'histoire culturelle. Ainsi peut-on assister, comme le suggère Luria, à l'intellectualisation des processus mémoriels élémentaires.

1. Conduites et conscience, II, p. 41-42.

2. Les jumeaux..., p. 33. 
$3 /$ Mais on l'a vu, l'influence des milieux varie en fonction de la réceptivité de l'organisme, de la structure de l'individu situé en face des structures complexes des milieux. La tâche du psychologue, s'opposant aux sociologismes réducteurs qui ne voient que le modelage des mentalités et des fonctions par les structures sociales, sera d'étudier les caractéristiques du psychisme qui permettent aux individus, non seulement d'être réceptifs aux milieux socioculturels, mais encore de les restructurer en fonction de leurs expériences, de leurs résistances, de leur créativité. Face aux régulations imposées par l'hérédité, face aux incitations des règles et des moeurs sociales, il y a une autonomie psychologique: «Elle consiste à briser l'esclavage des sollicitations du milieu. Non en les ignorant, mais en les chargeant du passé. » De telle sorte que la réponse pourra être différée, inhibée, négative, mais positive aussi quand le présent corrige le passé. L'autonomie est la maî" trise de l'instant présent, grâce à l'arbitrage du passé.

On peut avancer qu'une contribution essentielle de $\mathbf{R}$. Zazzo à la psychologie a été d'analyser l'architecture des processus psychologiques de l'autonomisation. Les philosophies spiritualistes font de celle-ci la mise en cuvre d'une structure a priori (ainsi de Kant). Les behavioristes voient en elle un processus d'adaptation au milieu, déterminé dans son devenir par les changements dans ce milieu, compte tenu de ceux qui proviennent de l'organisme. Zazzo veut reconnaître au psychisme une autonomie, dont il cherche les constituants premiers dans la relation à l'autre, les conditions de développement dans l'assouplissement continu des automatismes par la critique cognitive, dans un milieu social et devenir des modes anciens d'adaptation: dans une "création imprévisible de l'expérience» (Les jumeaux, p. 64-65).

On n'évoque que quelques aspects de cette théorie de l'autonomisation - chantier ouvert.

Quelques réflexions épistémologiques d'abord. Zazzo fait un examen critique de la notion d'adaptation au milieu social comme critère de l'intelligence. Les tests de Binet, et de ceux qui se sont inspirés de lui, partent de l'idée qu'il est possible d'évaluer les capacités d'adaptation d'un individu à son milieu social, sans tenir compte de l'évolution de ce milieu, en supposant qu'il existe des comportements qui sont l'indice de la maturité, et qu'il existe un modele d'adulte, d'une personnalité proche de la perfection, par rapport auquel on peut classer les individus. Zazzo estime qu'il y a une pluralité d'intelligences, que leur développement dépend des exercices des sujets dans les domaines correspondants (Les jumeaux, chap. II), qu'on ne peut conclure de l'existence de la maturation à l'existence d'un état de maturité. Le psychologue passe à côté d'une caractéristique des conduites humaines en mettant l'accent sur l'adaptation et la maturité: une part importante d'entre elles consiste à refuser l'intégration à l'entourage, à prendre conscience qu'elle exige le sacrifice de potentialités existantes, ou à susciter. Car on ne peut réduire l'entourage à ce qui est ici maintenant: «Le milieu de l'homme c'est un univers de projets et de valeurs, c'est l'avenir en germe dans le présent 》 (Des garçons, p. 365). 
Aussi, dans la construction de l'échelle de développement social, c'est en opposition à la notion de maturation que Zazzo se propose d'atteindre dans les conduites observables trois aspects du comportement qui soient des indicateurs - aux âges de 6 à 12 ans - de cette attitude novatrice : 1 / l'autonomie des conduites, l'attitude d'autogestion; 2/ l'intégration sociale, la socialité, en tant que disponibilité aux autres et participation active à des groupes divers; $3 /$ lintelligence sociale, comme curiosité pour les rapports sociaux et leurs ressorts (ibid., p. 321, 336-337).

Mais cette autonomie passe par un développement dont Zazzo a marqué les étapes dans sa recherche sur les jumeaux, dégageant par là la psychologie de l'enfant et de l'homme de l'emprise exercée sur elle par «les modèes animaux du comportement humain» aussi bien que par les modèles linguistiques et par ceux de la logique.

On peut placer au fondement de l'affirmation de la personne la conquete de la conscience de soi, dans sa singularité, dans son existence sociale, dans ses projets d'avenir, non seulement pour soi en tant qu'individu, mais aussi pour le soi homme. Sur la construction de ce qu'on peut appeler des modes humains d'exister, l'étude sur les jumeaux, celle aussi sur l'image de soi dans le miroir, fournissent des analyses décisives.

1/Les jumeaux identiques, élevés ensemble, en viennent assez rapidement à présenter des différences importantes dans leurs comportements. Ces asymétries se marquent notamment dans les traits de personnalité. Zazzo for mule l'hypothèse qu'il s'agit là d'un effet-de-couple: «Le couple est une structure où chacun des partenaires crée ses rôles en fonction des rôles de l'autre. Les différences de personnalité sont en bonne partie des effets-decouple» (Les jumeaux, 1991, p. 7). Zazzo se réfère à l'étude que fait Wallon, dans les Origines du caractère, du rôle assumé par l'autre dans la genèse du moi. Celui-ci, après une période de symbiose affective, en vient, grâce ì la maîtrise croissante de sa motricité, à des activités alternantes avec autrui. Il y apprend à devenir un centre d'initiatives, à provoquer les réactions de l'autre, à construire des imitations différées, à contrôler ses mouvements d'après son modèle. Ainsi se construit une socialité relativement indépendante des normes sociales, où chacun se perçoit comme distinct de son partenaire par sa capacité à susciter la réponse d'autrui. Chacun se distingue d'autrui en se rendant maître de ses gestes. Par la participation à une pluralité de couples (avec la mère, le père, les frères...) chacun use de son jeu avec l'un pour s'opposer à l'autre. Chez les jumeaux identiques, cette distinction peut être favorisée par des écarts sur le plan biologique, ou en fonction d'attitudes différenciées d'un éducateur en faveur de l'un des deux. Des asymétries se constituent sans que l'attachement entre les jumeaux se disloque, chacun cultive sa singularité, et en vient, surtout à partir de l'adolescence, à la revendiquer.

$2 /$ Ce n'est cependant pas le couple qui par lui seul procure l'autonomie. L'attachement réciproque des partenaires peut être nocif: chez les jumeaux on observe une communication dans un langage-jargon qui provoque retards linguistiques et intellectuels. Et ce n'est pas non plus par la vertu de la seule 
maturation, ou par celle des conditionnements, que se produisent les ouvertures successives à la conquête de l'autonomie. De ces ouvertures $R$. Zazzo fournit le principe général: «L'homme doit rompre tout à la fois sa sujétion affective au groupe et sa sujétion sensori-motrice; dépasser le règne animal et sortir du règne des opinions. Chacun des obstacles est un levier utilisé a la rupture de l'autre... La société nous arrache à la nature et la nature à la société », grâce au travail incessant de l'intelligence vers l'objectivité (Les jumeaux, p. 234). De ces arrachements aux stéréotypes d'activités constitués, solidifiés dans des cadres sociaux, Zazzo étudie l'enchainement dialectique dans des observations expérimentales d'une grande finesse, quantifiées, attentives à relever les étapes, leurs variations en fonction de situations bien définies (comparaisons entre vrais et faux jumeaux, comparaisons entre les âges et l'appartenance à des milieux socioculturels différents pour le développement psychosocial, dans Des garçons de 6 à 12 ans).

On note seulement quelques étapes de ces «ruptures» objectivées par R. Zazzo:

- ruptures avec les équilibres assurés sur le plan sensorimoteur et proprioceptif avec l'accès aux formes de symbolisme : conquête de la « reconnaissance » de soi dans l'image du miroir, qui est la découverte de son être comme - les autres, conscience d'un des dédoublements de soi qui vont permettre l'objectivation de soi. Ml n'aurait pas eu lieu sans le couple, et il permet d'en sortir. - Tous les déplacements des fictions assurent diverses objectivations du même ordre, qui ont notamment la fonction de projeter et de fonder le sujet en d'autres temps que le présent (Je voudrais être bébé/être grand, être lion, être sorcier, Le Bestiaire). Fallacieuses, les fictions délivrent des jeux sensori-moteurs;

- ruptures avec l'imaginaire (grâce au symbole et « contre » lui) avec le langage et les signes. Ils sont chargés de toutes les différenciations que les civilisations ont constituées, en traitant les activités sensori-motrices et les relations à autrui dans des pratiques qui visent l'accommodation aux propriétés des choses et des individus. Langage et intelligence ont partie liée, sans pourtant cöncider. Partie liée : car le premier est un instrument différenciateur et organisateur, mais l'intelligence a d'antres sources et d'autres fonctions, fondées dans des pratiques pour maitriser le réel (Les jumeaux, chap. IV).

3/Au-delà de ces ruptures vient l'autonomisation; elle est analysée par Zazzo sur le plan de la rupture d la sujétion affective aux groupes. Les croisements des couples y contribuent, mais dans la mesure où ils sont nourris de connaissances diverses. Il en est ainsi chez les jumeaux, qui s'arrachent à l'attachement premier qui les aliène, dans un travail d'individuation qui met en jeu aussi bien les incitations de la sexualité que les exigences de la division des tâches, les réussites supérieures de l'un ou l'autre dans certaines d'entre elles, les sentiments de dominance et d'infériorité, parfois encouragés par les milieux. Zazzo analyse ces sentiments et attitudes d'individuation sous une 
pluralité d'aspects (socialité, conflits dans l'accomplissement des rôles, le partage de l'affection, les rivalités qui peuvent aboutir au reniement de la gémellité). L'individuation n'est pas l'entreprise des seuls sujets. Elle est orienté par des représentations sociales qui l'encouragent et la répriment tour à tour, de façon différente dans les divers rôles reconnus au socius par les représentations sociales dominantes. Zazzo clinicien, dans les «témoignages parallèles》 qu'il tecuelle, relève des informations essentielles sur les cadres sociaux du travail de singularisation, de l'orientation par les groupes de la formation du sentiment d'identité. Ainsi, dans ces témoignages, au sujet des goûts, des cercles fréquentés, des opinions (chap. VI), voit-on affleurer linfluence des idéologies en conflit dans la société.

Or, on est ici dans le domaine que Zazzo et ses collègues aborderont à partir de 1960, dans des Garçons de 6 à 12 ans, par exemple à propos du dynamisme évolutif, de l'évolution des valeurs ou du développement social. Il s'agit bien de ce que Zazzo considère comme les attitudes qui préludent à la construction de la liberté dans l'affrontement par les sujets des problèmes de la société: des conflits qu'ils vivent dans la participation aux différents groupes de la société (quelle est leur valeur formative, quels sont les risques qu'ils font courir à l'avenir du développement..); de leur « intelligence sociale» (comment perçoiventils le devenir de la société, ses richesses et ses problèmes); de la stratégie enfin qu'ils élaborent pour se situer dans les contradictions de leur époque, des projets qu'ils construisent pour leur faire face.

René Zazzo souhaitait que la psychologie contribue à l'élaboration d'une connaissance scientifique de la liberté. 11 considérait que la tâche était difficile. Il enseigne qu'elle est possible et par quelles voies on peut l'entreprendre. Le psychologue peut-il avoir plus haute ambition? 